Revista Ibero-Americana de Ciências Ambientais

Ibero-American Journal of Environmental Science

Anais do Simpósio Interdisciplinar de Práticas de Produção

Agrícola e Conservação Ambiental - Jan 2017 - v.8 - n.1

ISSN: 2179-6858

\title{
Influência do congelamento na obtenção de blocos precursores de carvões adsorventes elaborados com bananas
}

O emprego de rejeitos da bananicultura na elaboração de materiais adsorventes, tem se apresentado favorável devido à diminuição dos custos de produção agregação de valor à matéria-prima, e aos bons resultados que vêm sendo registrados por diversos estudos de caso. $O$ objetivo deste trabalho foi caracterizar carvões adsorventes elaborados a partir de bananas verdes e maduras, empregando-se cascas, polpa e os frutos integrais, considerando duas metodologias que envolveram ou não, o congelamento como uma das etapas de obtenção dos blocos precursores dos carvões adsorventes. Foram avaliadas as propriedades físicoquímicas dos carvões, sendo que não foram obtidas diferenças significativas nas variáveis umidade e rendimento em massa, no que se refere ao emprego do congelamento como uma das etapas de confecção dos blocos. Os carvões feitos a partir das cascas de bananas verdes e maduras apresentaram menor teor de cinzas quando não se empregou o congelamento. Os blocos feitos com cascas de banana verde e com a polpa de banana madura apresentaram maior densidade aparente quando se utilizou o congelamento. Apenas a densidade relativa dos blocos feitos com polpa de bananas maduras apresentou diferenças, em que o congelamento influenciou na redução do valor deste parâmetro. A porosidade total dos adsorventes foi maior quando o congelamento não foi utilizado na elaboração da maioria dos tipos de blocos. Desta forma, o emprego do congelamento se mostrou importante para promover a aglutinação da massa feita com bananas, conferindo assim, o formato e a compactação dos blocos até o final do processamento, entretanto, esta etapa não exerceu relevância frente às características físico-químicas avaliadas.

Palavras-chave: Reaproveitamento; Musa spp., Carbonáceo; Adsorção.

\section{Influence of freezing in the obtainment of adsorbent carbon precursor blocks made with bananas}

\begin{abstract}
The use of residues from banana cultivation in the elaboration of adsorbent materials has presented itself favorable due to the decrease of production costs, and also because it provides value to the raw material, and helps to achieve good results, which have been registered by several case studies. The aim of this work was tocharacterize adsorbents carbons elaborated from green and ripe bananas, employing peels, pulp, and whole-grain fruits. For that, it was considered two methodologies that involved or not the freezing as one of the stages in the obtaining of precursor blocks of adsorbent carbons. The physicochemical properties of carbons were evaluated, showing that there were no significant differences in the variables moisture and mass yield, with regard to the use of freezing as one of the stages of blocks confection. Carbons made from green and ripe bananas peels presented lower ash content when it was not employed the freezing. The blocks made with green bananapeels and ripe banana pulp presented more relative density when it was used the freezing. Only the relative density of the blocks made with ripe bananaspulp has presented differences, in which the freezing influenced in the decrease of value of this parameter. Theadsorbents total porosity was larger when it was not used the freezing in the elaboration of the majority of blocks types. Therefore, the use of the freezing proved to be important to promote the agglutination of the mass made with bananas, thus, giving the format and the compaction of the blocks until the end of processing. However, this stage was not relevant, in view of the evaluated physicochemical characteristics.
\end{abstract}

Keywords: Reutilization; Musa SPP.; Carbonaceous; Adsorption.

Topic: Química Agrícola e Ambiental

Reviewed anonymously in the process of blind peer.

Pércia Graczyk de Souza

Universidade do Estado de Mato Grosso, Brasil

http://lattes.cnpq.br/2933690731078220

graczykpercia@gmail.com

Tadeu Miranda de Queiroz

Universidade do Estado de Mato Grosso, Brasil

http://lattes.cnpq.br/2582121765769124

tdmqueiroz@yahoo.com.br

Thaynara Melo Yamaguchi

Universidade do Estado de Mato Grosso, Brasil

http://lattes.cnpq.br/5410885448507292

thay gif@hotmail.com

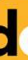

DOI: 10.6008/SPC2179-6858.2017.001.0014
Received: 18/04/2016

Approved: 20/05/2016
Referencing this:

SOUZA, P. G.; QUEIROZ, T. M.; YAMAGUCHI, T. M.. Influência do congelamento na obtenção de blocos precursores de carvões adsorventes elaborados com bananas. Revista Ibero-Americana de Ciências Ambientais, v.8, n.1, p.162-170, 2017. DOI:

http://doi.org/10.6008/SPC2179-6858.2017.001.0014 


\section{INTRODUÇÃO}

De acordo com a Organização das Nações Unidas para Alimentação e Agricultura (FAO) são desperdiçados 1,3 milhões de toneladas de alimentos no mundo, causando uma perda econômica de 750 bilhões de dólares anualmente (FAO, 2013). A perda de produção agrícola é capaz de gerar impactos profundos ao meio ambiente, relacionados principalmente a disponibilidade de água para irrigação, terras cultiváveis, áreas pesqueiras, e aos excessos de consumo de energia (FAO, 2015).

Dados expressivos sobre o desperdício alimentar no Brasil, o colocam como um dos países que mais desperdiçam comida no mundo, embora não existam valores conclusivos a respeito do assunto. Segundo Soares (2014), o problema ganha destaque na produção agrícola, onde há falhas nas etapas de produção, manipulação pré e pós-colheita, armazenamento, distribuição e consumo.

É o caso da bananicultura, importante atividade agrícola amplamente praticada por produtores rurais brasileiros, onde são registradas perdas da produção de banana, devido às doenças e ao conjunto de problemas fitotécnicos. Além disso, a falta de cuidados na distribuição e comercialização provoca injúrias nos frutos e dificulta a apreciação pelos consumidores (GASPAROTTO et al., 2003).

Com a série de ações desenvolvidas no intuito de minimizar os impactos ambientais causados pelos descartes indevidos de resíduos orgânicos, realizam-se estudos que buscam reaproveitar e/ou utilizar frutos da bananeira, ou parte destes, na elaboração de: produtos alimentícios (STORCK et al., 2013), compostagem (FERREIRA et al., 2015), materiais adsorventes (MARTINS et al., 2015), entre outros.

O emprego de carvão ativo no tratamento de água para consumo e de efluentes industriais é uma das tecnologias mais importantes envolvendo a adsorção (NAJM et al., 1991). Desta forma, a elaboração de produtos com capacidade adsortiva a partir dos rejeitos da bananeira apresenta potencial, já que se alia a diminuição dos gastos de produção e agrega valor a matéria-prima.

Somam-se a isto, os bons resultados obtidos na remoção de corantes utilizando pseudocaule da bananeira (SILVA et al., 2010) e de metais pesados adsorvidos em cascas de banana (BONIOLO et al., 2010). No entanto, as metodologias aplicadas na elaboração de carvões adsorventes a partir de rejeitos da atividade agrícola se trata de estudos de caso, onde as propriedades físico-químicas dos produtos adquiridos podem variar de acordo com o material precursor utilizado.

Neste trabalho, foram aplicados dois diferentes métodos de elaboração de blocos precursores de carvões adsorventes, feitos a partir de bananas verdes e maduras, empregando-se cascas, polpa, e cascas com polpa (frutos integrais). Trata-se de metodologias que utilizam ou não o congelamento como a etapa de aglutinação da massa de banana, a fim de transformá-la em blocos, e assim, o objetivo foi avaliar as propriedades físico-químicas dos carvões adsorventes gerados a partir dos blocos precursores desenvolvidos. 


\section{METODOLOGIA}

\section{Matéria-Prima Empregada e Preparação do Material Precursor}

Os carvões adsorventes foram preparados a partir de bananas (Musa spp.) da variedade Terra, adquiridas na feira livre municipal da cidade de Barra do Bugres- MT. As bananas utilizadas foram selecionadas de acordo com a escala de Von Loesecke (1950), nos estágios de maturação totalmente verde e em senescência, foram higienizadas para a remoção das sujidades e imersas em água fervente, onde permaneceram sob pressão por 15 minutos.

Após a cocção, as bananas foram liquidificadas separando-as nas proporções de massas feitas com cascas, polpa e cascas com polpa (fruto integral). As massas obtidas foram moldadas com auxílio de moldes cilíndricos de PVC e divididas em duas parcelas, onde uma delas foi submetida diretamente ao processo de secagem em estufa (105으) por 24 horas, e a outra, previamente submetida ao congelamento a fim do aglutinamento da massa, através do emprego do frio.

Quadro1: Descrição das denominações atribuídas aos tipos de materiais precursores obtidos através dos processos com e sem congelamento.

\begin{tabular}{|c|c|c|c|}
\hline Tipo do processo & Estágio de maturação & Composição & Denominação \\
\hline \multirow{6}{*}{ Com congelamento } & \multirow{3}{*}{ Verde } & Casca & CVC \\
\hline & & Polpa & CVP \\
\hline & & Integral & $\mathrm{CVI}$ \\
\hline & \multirow{3}{*}{ Madura } & Casca & $\mathrm{CMC}$ \\
\hline & & Polpa & CMP \\
\hline & & Integral & $\mathrm{CMI}$ \\
\hline \multirow{6}{*}{ Sem congelamento } & \multirow{3}{*}{ Verde } & Casca & SVC \\
\hline & & Polpa & SVP \\
\hline & & Integral & SVI \\
\hline & \multirow{3}{*}{ Madura } & Casca & SMC \\
\hline & & Polpa & SMP \\
\hline & & Integral & SMI \\
\hline
\end{tabular}

\section{Ativação Química e Carbonização}

A carbonização foi precedida da etapa de ativação química, em que os materiais precursores foram imersos em solução de $\mathrm{KOH} 10 \%$ por 1 hora, e secos em estufa a 105ㄷ․ Assim, as amostras foram submetidas à carbonização em mufla $(550$ 으 $)$ durante 15 minutos. Posterior a este processo, os carvões obtidos foram tratados com solução de $\mathrm{HCl} 10 \%$ por 2 horas, e por fim, secos em estufa (105ㅇ) por 24 horas (Adaptado de SUGUMARAN et al., 2012).

\section{Caracterização Físico-Química dos Carvões Adsorventes}

\section{Densidade aparente, relativa e porosidade total}

De acordo com Embrapa (1997), a densidade relativa foi obtida pesando cerca de 1 grama de amostra previamente seca em estufa a $105^{\circ} \mathrm{C}$ por 12 horas, em balão volumétrico de $25 \mathrm{ml}$, para que fosse aferido o seu volume com álcool etílico $96 \%$. E a densidade aparente, foi determinada utilizando a metodologia do 
torrão parafinado. Feito isso, a porosidade total do carvão foi obtida a partir dos resultados da densidade relativa e aparente, utilizando a equação:

$$
P(\%)=\left(1-\frac{D a}{D r}\right) \times 100
$$

Em que,

$$
P(\%)=\text { Porosidade total, \%; }
$$

$D a=$ Densidade aparente, $\mathrm{g} / \mathrm{cm}^{3}$;

$D r=$ Densidade relativa, $\mathrm{g} / \mathrm{cm}^{3}$.

\section{Umidade}

A umidade dos carvões foi determinada conforme a norma ABNT MB - 3414 (1991), pesando as amostras em placas de Petri previamente secas e taradas, onde posteriormente foram submetidas à secagem em estufa (130ㄷ) por 3 horas. Após anotar o valor da massa de carvão seco, a umidade foi dada pela seguinte equação:

$$
\begin{array}{r}
U(\%)=\frac{P_{1}-P_{2}}{P_{1}} \times 100 \\
U(\%)=\text { Umidade em massa de amostra úmida, \%; } \\
P_{1}=\text { Massa da amostra úmida, } \mathrm{g} ; \\
P_{2}=\text { Massa da amostra seca, } \mathrm{g} .
\end{array}
$$

\section{Cinzas}

Para a análise do teor de cinzas, as amostras foram levadas à mufla $(550 \circ \mathrm{C})$ onde permaneceram por no mínimo 12 horas, até sua calcinação completa (JIS, 1992). 0 teor de cinzas foi obtido através da equação:

$$
\begin{array}{r}
C(\%)=\left(\frac{M c}{C s}\right) \times 100 \\
C(\%)=\text { Em que, } \% ; \\
M c=\text { Massa de cinzas, g; } \\
C s=\text { Massa da amostra previamente seca, } \mathrm{g} .
\end{array}
$$

\section{Rendimento em massa}

A determinação do rendimento em massa foi realizada de acordo com loannidou e Zabaniotou (2007), em que os blocos foram pesados antes e após a carbonização. Feito isso, o rendimento foi obtido pela equação:

$$
R(\%)=\left(\frac{M c}{M a}\right) \times 100
$$

Em que,

$$
\begin{array}{r}
R(\%)=\text { Rendimento em massa após a carbonização, \%; } \\
M c=\text { Massa do bloco carbonizado, g; } \\
M a=\text { Massa do bloco precursor, g. }
\end{array}
$$




\section{Análise Estatística}

Anormalidade foi verificada pelo $p$-valor $>0,05$, considerando seis grupos de dados independentes (VC, VP, VI, MC, MP e MI), e assim, foi constatada a relação paramétrica. Para verificar a influência do congelamento sobre os diferentes tipos de amostras, utilizou-se o experimento inteiramente casualizado, com três repetições para cada metodologia de obtenção dos blocos precursores utilizados: com congelamento e sem. Os resultados foram submetidos à análise de variância (ANOVA), e quando verificado diferenças significativas, foram aplicados no teste Tukey ao nível de $5 \%$ de probabilidade utilizando o Software Assistat7.7 beta versão livre.

\section{RESULTADOS E DISCUSSÃO}

Os materiais precursores elaborados com a etapa de congelamento possuem características físicas mais bem definidas (Figura 1), pois os moldes adquirem formato cilíndrico rígido que são mantidos após a secagem, com exceção apenas da redução de tamanho devido à perda de umidade.

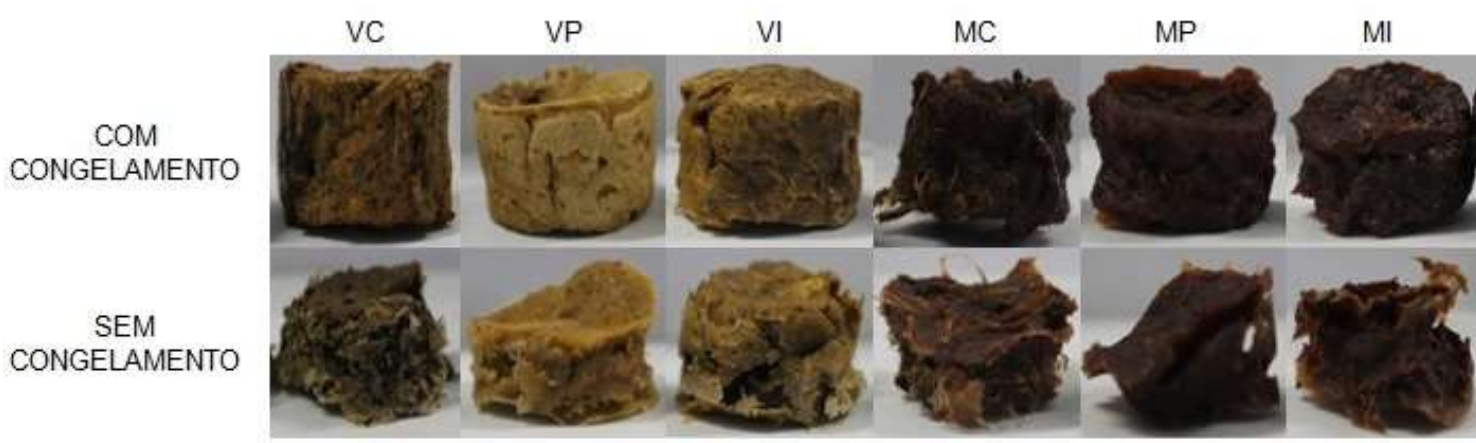

Figura 1: Materiais precursores obtidos de bananas verdes e maduras, nas composições de cascas, polpa e cascas com polpa (integral) através de métodos que envolveram o congelamento ou não.

Na Tabela 2 são apresentados os resultados obtidos nas determinações físico-químicas dos carvões adsorventes, onde os valores de umidade e rendimento não demonstraram diferenças significativas, quanto ao tipo de metodologia aplicada para a obtenção dos materiais precursores, elaborados com bananas verdes ou maduras, quando submetidos à análise de variância.

Tabela 2: Valores médios obtidos nas determinações de umidade (U), cinzas (C), rendimento (R), densidade aparente (Da), densidade relativa ( $\mathrm{Dr}$ ) e porosidade total $(\mathrm{P})$ dos carvões adsorventes desenvolvidos a partir de bananas verdes e maduras.

\begin{tabular}{|c|c|c|c|c|c|c|c|c|}
\hline \multirow{2}{*}{\multicolumn{3}{|c|}{ Carvões }} & U & C & $R$ & $\mathrm{Da}$ & $\mathrm{Dr}$ & \multirow{2}{*}{\begin{tabular}{c|}
$\mathrm{P}$ \\
$(\%)$ \\
\end{tabular}} \\
\hline & & & \multicolumn{3}{|c|}{ (\%) } & \multicolumn{2}{|c|}{$\left(\mathrm{g} / \mathrm{cm}^{3}\right)$} & \\
\hline \multirow{6}{*}{ Verde } & \multirow{2}{*}{ Casca } & CVC & $4,22 a$ & $12,77 a$ & $37,04 a$ & $0,07 a$ & $0,35 a$ & $79,46 b$ \\
\hline & & SVC & $4,76 a$ & $8,18 \mathrm{~b}$ & $34,56 a$ & $0,05 b$ & $0,31 a$ & $84,44 a$ \\
\hline & \multirow{2}{*}{ Polpa } & CVP & $4,85 a$ & $7,10 a$ & $33,13 a$ & $0,15 a$ & $0,40 a$ & $61,89 a$ \\
\hline & & SVP & $4,80 a$ & $6,25 a$ & $33,37 a$ & $0,16 a$ & $0,40 a$ & $59,03 a$ \\
\hline & \multirow{2}{*}{ Integral } & $\mathrm{CVI}$ & $6,10 a$ & $8,25 a$ & $35,45 a$ & $0,15 b$ & $0,39 a$ & $60,86 a$ \\
\hline & & SVI & $5,07 a$ & $6,97 a$ & $35,52 a$ & $0,23 a$ & $0,45 a$ & $49,38 b$ \\
\hline \multirow{6}{*}{ Madura } & \multirow{2}{*}{ Casca } & CMC & $5,11 a$ & $14,42 a$ & $28,78 a$ & $0,08 a$ & $0,26 a$ & $67,70 b$ \\
\hline & & SMC & $4,56 a$ & $11,01 b$ & $25,92 a$ & $0,07 a$ & $0,33 a$ & $78,22 a$ \\
\hline & \multirow{2}{*}{ Polpa } & CMP & $5,75 a$ & $5,48 a$ & $24,85 a$ & $0,31 a$ & $0,72 b$ & $56,99 b$ \\
\hline & & SMP & $4,41 a$ & $6,41 a$ & $23,41 a$ & $0,16 b$ & $0,83 a$ & $80,42 a$ \\
\hline & \multirow{2}{*}{ Integral } & $\mathrm{CMI}$ & $6,07 a$ & $9,93 a$ & $24,43 a$ & $0,24 a$ & $0,66 a$ & $63,35 b$ \\
\hline & & SMI & $5,71 \mathrm{a}$ & $10,98 a$ & $25,76 a$ & $0,17 a$ & $0,69 a$ & $75,47 a$ \\
\hline
\end{tabular}

Médias seguidas da mesma letra na coluna para a mesma variável e composição do material precursor, não apresentam diferenças significativas a 5\% de probabilidade pelo teste de Tukey. 
Os valores obtidos na determinação da umidade dos carvões (Tabela 2), está relacionado com as condições de armazenamento ou ambiente a qual os mesmos são expostos. Todas as amostras elaboradas neste estudo eram acondicionadas em sacos plásticos codificados, para evitar que os carvões absorvessem umidade do ar. Por isso, não há variações que se associem ao emprego do congelamento sob qualquer um dos tipos de carvões.

Cabe ressaltar, que de acordo com a norma EB-2133 para o fornecimento de carvão ativado pulverizado, o teor de umidade não deve ultrapassar 8\% (ABNT, 1991), demonstrando que os valores encontrados para essa variável estão dentro dos padrões exigidos para comercialização.

Quanto aos dados obtidos na análise de rendimento (Tabela 2), pode-se observar que não houve diferenças significativas entre os carvões elaborados com a etapa de congelamento envolvida no processo ou não. Embora o estudo não tenha tido o objetivo de abordar sobre as diferenças entre as composições dos carvões, observa-se a influência do estágio de maturação das bananas sob este parâmetro, em que os frutos maduros tendem a um menor rendimento devido ao maior teor de açúcar dos blocos precursores, que por consequência da carbonização se volatilizam com mais facilidade, da mesma forma que os blocos elaborados com polpa em sua composição.

De acordo com loannidou e Zabaniotou (2007), o rendimento é um fator capaz de avaliar a quantidade de carbono consumido durante o processo de pirólise, e tem relação com constituição porosa dos carvões. Em estudos de Costa (2007), foi verificado que valores maiores de rendimento pressupõem a formação de poros com diâmetros maiores, logo, quando o rendimento se apresenta menor que 50\% possivelmente há formação de microporos, o que favorece a adsorção de moléculas pequenas de adsorvato, tal como, metais pesados.

Os valores de cinzas apresentaram diferenças significativas apenas para os carvões elaborados com as cascas das bananas, sendo estas verdes ou maduras (Tabela 2), e segundo Mucciacito (2006), o teor de cinzas depende da composição da matéria prima e do processo de fabricação que o carvão foi submetido. Assim, é possível que o tempo e a temperatura de carbonização padronizada para todos os tipos de amostras, tenha sido insuficiente para tornar os blocos precursores elaborados com a etapa de congelamento, em materiais carbonáceos com baixo teor de cinzas.

As cinzas são compostos inorgânicos que não sofrem carbonização (KHEZAMI et al., 2007), e quando apresentados em grandes quantidades, podem desfavorecer a adsorção do adsorvato de interesse (MORENO-CASTILLA, 2004). Por isso, as amostras SVC e SMC se mostraram mais favoráveis para este fim, já que se observam diferenças entre as metodologias de sua elaboração.

Os valores médios encontrados para a densidade aparentem dos carvões se diferenciaram significativamente entre as amostras: CVC e SVC, CVI e SVI e CMP e SMP (Tabela 2). Observa-se que a amostra CVI apresentou menor densidade aparente, diferentemente do SVC e SMP, que se mostraram menos densos sem o emprego da etapa de congelamento.

Sugumaranet al. (2012), afirmam que a densidade aparente é uma das características importantes a ser avaliada, pois depende do material precursor utilizado e do método de elaboração dos carvões. Além 
disso, determina-se este parâmetro considerando os espaços vazios no interior dos blocos, então, quanto menor a densidade aparente maior a abertura de poros deste material, em que possivelmente poderá contribuir para um melhor desempenho do mesmo em ensaios de adsorção.

Na determinação da densidade relativa, não são considerados os espaços vazios contidos no interior dos carvões adsorventes, por isso, os valores tendem a serem superiores aos encontrados para a densidade aparente. As diferenças foram encontradas entre as amostras CMP e SMP, em que os blocos elaborados na ausência da etapa de congelamento se mostraram mais densos, entretanto, o valor é favorável devido a sua relação com a densidade aparente, fazendo com que a porosidade total também se apresente maior quando comparada aos outros carvões (Tabela 2).

Foi encontrado por Sugumaranet al. (2012), o valor de $0,34 \mathrm{~g} / \mathrm{cm}^{3}$ para a densidade relativa de um carvão ativado com $\mathrm{KOH}$ feito a partir do pseudocaule da bananeira, corroborando com os resultados encontrados neste estudo em materiais elaborados com cascas de bananas verdes ou maduras. Isso se deve a característica fibrosa de ambos, que conferem aos adsorventes mais espaços vazios em seu interior, e consequentemente, menor densidade do que aqueles confeccionados com a polpa do fruto.

Os dados obtidos na determinação da porosidade total se diferenciaram significativamente para quase todas as amostras (Tabela 2). Para o bloco de carvão do tipo SVC verificou-se que a etapa de congelamento não se mostrou importante para a atribuição de porosidade ao material, contraditoriamente ao CVI que tem em sua composição a polpa de banana verde.

Conforme Valle e Camargos (2002), a polpa banana verde possui uma composição rica em amido, que através do processo de amadurecimento se transformam em açúcares. A partir do pressuposto, é provável que o emprego de baixas temperaturas aos materiais precursores dos blocos de carvão, faz com que o arranjo das partículas de cada composição elaborada, se comporte de forma diferente, promovendo mais ou menos porosidade ao bloco.

Já os blocos dos tipos SMC, SMP e SMI apresentaram maior porosidade, quando não foram submetidos ao processo de congelamento durante as etapas de confecção dos materiais precursores. Segundo Yan et. al. (1996), a porosidade favorece a adsorção e a densidade está relacionada à força mecânica dos blocos, desta forma, à maior porosidade em conjunto com a maior densidade relativa atribuída a estes materiais, pode viabilizar seu uso em processos adsortivos que utilizam alto fluxo de determinados fluidos.

Os carvões elaborados com os materiais precursores do tipo VP não apresentaram diferenças diante as densidades aparente e relativa, e porosidade total, possivelmente em virtude da desintegração no momento da ativação química, e posterior, aglomeração irregular durante o processo de carbonização. Isso faz com que os blocos deixem de assumir o formato inicial de bloco, adquirido com ou sem congelamento, tornando sua característica física desconforme independentemente do método utilizado.

\section{CONCLUSÕES}

O congelamento se mostrou importante para a formação dos blocos dos carvões adsorventes elaborados a partir das bananas verdes e maduras, pois além de facilitar o manuseio da massa obtida antes 
da etapa de secagem, favoreceu aos mesmos a compactação necessária para que permanecessem com este formato até o final do processamento, com exceção apenas dos materiais compostos pela polpa de banana verde.

O emprego de baixas temperaturas aos blocos precursores, não produziu diferenças significativas às características físico-químicas da maioria dos tipos de carvões obtidos, o que pode tornar esta metodologia inviável.

\section{AGRADECIMENTOS}

Os autores agradecem os Laboratórios de Qualidade de Água (LaQuA), de Química Geral e Experimental, de Engenharia de Produção Agroindustrial e de Matérias-Primas para a Produção de Biodiesel da Universidade do Estado de Mato Grosso (UNEMAT), Campus de Barra do Bugres por disponibilizar seus equipamentos e oportunizar a realização desta pesquisa, como também, a CAPES e FAPEMAT pela concessão de bolsa de mestrado.

\section{REFERÊNCIAS}

ABNT. Associação Brasileira de Normas Técnicas. NBR 12077 MB 3414 Carvão ativado pulverizado: Determinação da umidade. Rio de janeiro: ABNT, 1991.

BONIOLO, M. R.; YAMAURA, M; MONTEIRO, R. A.. Biomassa residual para remoção de íons uranilo. Revista Química Nova, São Paulo, v.33, n.3, p.546-541, 2010.

COSTA, L. C. M.. Produção e modificação de carvões ativados para aplicações ambientais. (Doutorado em Ciências - Química) - Universidade Federal de Minas Gerais, Belo Horizonte, 2007. DOI: http://doi.org/10.14583/23187670.v03n02a08

EMBRAPA. Empresa Brasileira de Pesquisa Agropecuária. Manual de métodos de análise de solo. 2 ed. Rio de Janeiro, 1997.

FAO. Food and Agriculture Organization of the United Nations.Food wastage footprint: Impacts on natural resources, 2013.

FAO. Food and Agriculture Organization of the United Nations.OECD-FAO Agricultural Outlook 2015-2024. Paris: OECD Publishing, 2015.

FERREIRA, M. C.; COSTA, S. M. L.; PASIN, L. A. A.. Uso de resíduos da agroindústria de bananas na composição de substratos para produção de mudas de pau pereira. Nativa, Sinop, v.3, n.2, p.120-124, 2015.

GASPAROTTO, L.; PEREIRA, J. C. R.; PEREIRA, M. C. N.. Manejo Integrado de Doenças da Bananeira. Circular Técnica 19. Manaus: EMBRAPA, 2003.

IOANNIDOU, O; ZABANOTOU, A.. Agricultural residues as precursors for activated carbon production - A review. Renewable and Sustainable Energy Reviews, v.11, p.19662005, 2007. DOI: http://doi.org/10.1016/j.rser.2006.03.013
JIS. Japanese Industrial Standard. Activated carbons, powdered and granular - Methods of sampling and test. 2 ed. New Delhi: BIS, 1992.

KHEZAMI, L.; OULD-DRIS, A.; CAPART, R.. Activated carbon from thermo-compressed wood and other lignocellulosic precursors. BioResources, Raleigh, v.2, n.2, p.193-209, 2007.

MARTINS, W. A.; OLIVEIRA, A. M. B. M.; MORAIS, C. E. P.; COELHO, L. F. O.; MEDEIROS, J. F.. Reaproveitamento de resíduos agroindustriais de casca banana para tratamento de efluentes. Revista Verde de Agroecologia e

Desenvolvimento Sustentável, Pombal, v.10, n.1, p.96-102, 2015.

MORENO-CASTILLA, C.. Adsorption of organic molecules from aqueous solutions on carbon materials. Carbon, v.42, p.83-94, 2004. DOI:

http://doi.org/10.1016/j.carbon.2003.09.022

MUCCIACITO, J. C.. Conceitos e aplicações do carvão ativado. Revista Meio Filtrante, São Paulo, v.22, 2006.

NAJM, I. N.; SNOEYINK, V. L.; LYKINS JÚNIOR, B. W.; ADAMS J. Q.. Using Powdered Activated Carbon: A Critical Review. Research \& Technology, p.65-76, 1991.

SILVA, F. M.; SANTANA, S. A. A.; BEZERRA, C. W. B.; SILVA, H. A. S.. Adsorção do corante têxtil azul de remazol $R$ por pseudocaule da bananeira (musa sp). Cadernos de Pesquisa, São Luís, v.17, n.3, p.71-77, 2010.

SOARES, A. G.. Desperdício de Alimentos no Brasil: um desafio político e social a ser vencido. Rio de Janeiro: EMBRAPA, 2014.

STORCK, C. R.; NUNES, G. L.; OLIVEIRA, B. B.; BASSO, C.. Folhas, talos, cascas e sementes de vegetais: composição nutricional, aproveitamento na alimentação e análise 
sensorial de preparações. Revista Ciência Rural, Santa Maria, v.43, n.3, p.537-543, 2013.

SUGUMARAN, P.; SUSAN, V. P.; RAVICHANDRAN, P.; SESHADRI, S.. Production and characterization of activated carbon from banana empty fruit bunch and Delonixregiafruit pod. Journal of Sustainable Energy \& Environment Bangkok, v.3, p.125-132, 2012.
VALLE, H. F.; CAMARGOS, M.. Yes, nós temos BANANAS: Histórias e receitas com biomassa de banana verde. São Paulo: Senac, 2002.

VON LOESECKE, H. W.. Bananas: Chemistry, Physiology, Technology. 2 ed. Washington: Interscience, 1950.

YAN, Z. Q.; McCUE, J. C.; TOLLES, E. D.. Preparation of high activity, high density activated carbon with activatable binder. 1996. 